\title{
Einstein: Unification Theory and Unification of Basic Forces-III
}

\author{
M. Tajuddin ${ }^{1}$, Md. Haider Ali Biswas ${ }^{2}$, \\ ${ }^{1}$ Directorate of Primary Education, Dhaka, Bangladesh \\ ${ }^{2}$ Mathematics Discipline, Khulna University, Khulna, Bangladesh
}

Email address:

dmtajuddin@yahoo.com (M. Tajuddin), mhabiswas@yahoo.com (M. H. A. Biswas)

\section{To cite this article:}

M. Tajuddin, Md. Haider Ali Biswas. Einstein: Unification Theory and Unification of Basic Forces-III. American Journal of Physics and Applications. Vol. 3, No. 6, 2015, pp. 190-202. doi: 10.11648/j.ajpa.20150306.12

\begin{abstract}
The bondage between the long range elementary forces and the short range three planetary forces is called unified force. 'Every particle feels the force of gravity according to its mass or energy' (Hawking, 1996). 'So the long range forces have appeal to the short range three planetary forces in inertia mass of the earth. The long range virtual particles discharging from the sun induce to the three matter forces of inertia of mass of the earth and the short range three matter forces sustain that force in their own way and thus results in unified force'-(Tajuddin and Biswas, 2015). 'All the known particles in the universe can be divided into two groups: particles of spin $1 / 2$, which make up the matter in the universe, and particles of spin 0,1 , and 2 , which we shall see, give rise to forces between the matter particles' ( Hawking, 1996). This unified force 'that will describe everything in the universe' (Hawking, 1996). But unified force will not combine all the partial theories of physics but it will bring all motions under the banner of unified force and spherical geometry as all motions are spherical in nature and all motions are alike. Thus motions have uniformity of spherical nature that creates unified force in all respect which is the ultimate goal of unification theory and unified force. (Vide: Present and previous articles published in AJPA).
\end{abstract}

Keywords: Unified Force, Interaction of Two Groups, Principles of Four, Nuclear Fission and Fusion

\section{Introduction}

Mankind knocks the door of super physics which is beyond the domain of nuclear physics. 'Nuclear physics which deals with nuclei and release of atomic energy' but super physics deals with structure less elementary particles that constitute stars. Nuclear physics deals with more or less structured particles (Vide: Composition of stars). The word nuclear derives from the word nucleus that remains in the centre of atom. The function of nuclear force is to bind particles in atoms. So the domain of nuclear physics may not go beyond the periphery of nucleus. 'The strong nuclear force has a curious property called confinement: it always binds particles together into combination' (Hawking, 1996). We cannot call elementary forces as nuclear force because they do not belong to nuclear bindings in atom such as they primarily interact with three matter forces and the matter forces secondarily interact with themselves through elementary forces. The elementary forces do not work in the same way as that of nuclear forces. 'All the known particles in the universe can be divided into two groups: particles of spin $1 / 2$ which make up the matter in the universe, and particles of spin 0,1 and 2, which we shall see, give rise to the forces between the matter particles' (Hawking, 1996). All particles in general may be classified into two groups according to their nature of works in different terms such as long range particles and short range particles, elementary particles and matter particles, long range spin $1 / 2$ and $1 / 3$, and short range spin 0,1 and 2 particles, making up matter particles and binding particles. These groups may also be termed adding force such as long range forces and short range forces, elementary forces and matter forces, elementary forces and planetary forces etc. Both the group termed as energy also. (Vide: Principles of four). Four categories of forces of two groups have different types of origins, developments and interactions. Long range particles come from the distance but short rage particles remain in the inertia of mass of planets. Elementary particles are virtual particles which are indivisible but the matter particles are divisible. The stars are conceived as the burning store of elementary particles from which it discharges elementary particles all over the world and control the universe. The planets gradually develop the matter particles in support of elementary particles. The bondage of the two 
groups creates unified force (Vide: Elementary forces and matter forces). 'Every particle feels the force of gravity according to its mass or energy' (Hawking, 1996). 'So the long range forces have appeal to the short range three forces lying in inertia of mass of the earth. The long range virtual particles discharging from the sun induce to the three planetary forces of inertia of mass of the earth and the short range forces sustain that force and thus results in unified force. Here gravitation and its allied lepton group virtual particles discharging from the sun and other heavenly bodies are known as long range particles that interact with the short range forces belong to hadrons group lying in the inertia of mass of the earth and other planets, and create unified force that controls all activities and all motions of the universe spherically. The bondage of the two groups creates unified force and unless there is bondage gravitation may not come to meet the opposite hadrons group and the universe will not work. Lepton group particles are not charge less as it is considered. Here induction of the two groups of forces is important' (Tajuddin and Biswas, 2015).

\section{Unified Force}

Einstein framed law of gravitation where a small particle moves around the sun in empty space. 'Einstein wished to relate all the properties of matter in a unified field theory' (Strathern, 1997). Einstein found that space-time is curved and gravitation is also curved and they curve all motions. Again in quantum mechanics 'Einstein's main scientific interest was in developing a unified theory, an attempt to explain both gravity and electromagnetism in one mathematical package' (White and Gribbin, 2005). But Einstein could not succeed in both the attempts because we know from his system of approach to metric, and Schwarzschild solutions that he used Euclidean type of zero tensors in his metric. We have strong observation that the earth and other planets move around the sun. We also observed using modern telescopes that all galaxies have spiral motion. As the galaxies have spiral motion, the solar system has spherical motion and all motions are alike. Thus unified force includes all motions and follows the principles of mechanical advantages.

What is unified force? The bondage between long range elementary forces and short range three planetary forces creates unified force that controls the universe and all motions. 'The sun has rotational and orbital movements and as a result the long range virtual particles discharged from the sun induce spherically to short range three forces such as electromagnetic, weak and strong nuclear forces lying in the inertia of mass of the earth. 'Every particle feels the force of gravity according to its mass or energy' (Hawking, 1996). So the long range forces have appeal to the short range three forces in inertial mass of the earth. The long range virtual particles discharging from the sun induce to three planetary forces lying in the inertia of mass of the earth and the short range three forces sustain that force in their own way and thus results in unified force.

The earth with its vast inertial mass rotates on its own axis and also around the sun due to spherical attraction of unified force. Thus unified force is formed by four categories of forces with some extra properties of matter such as radius of circle, cyclic frequency, mass of the body that controls the universe. (Tajuddin and Biswas, 2015).

\section{How the Universe Is Working}

The universe has ultimate principle known as unified force on which the universe is working. The unified force is deep rooted to the formation of the universe (Vide: Compositions of stars). The elementary particles spherically discharged from the distance, interact with three matter forces such as electromagnetic, weak and strong nuclear forces lying in the inertia of masses of the planets and create unified force that controls the solar system and the same type of greater unified force controls the universe. After big bang the universe scattered away and the breaking away parts began to move spherical way to keep a linkage among them. Galaxies use to move in spiral motion and planets move around the stars. Thus all motions are spherical in nature and all motions are alike. This is the reason 'the universe looks identical in whatever direction we look' (Hawking, 1996). Thus the universe is not chaotic but cosmos. As electricity derives from electromagnetic force, in the same way three matter forces derive from unified force where the unity lies.

Elementary forces are the basic forces of the universe lying in the stars and matter forces are the secondary forces known as planetary forces lying in the planets. These two groups interact in two ways such as elementary forces to three matter forces and elementary forces to elementary forces and create unified forces. First category of unified force controls the solar system in spherical motion and second category controls the galaxy system in spiral motion. This interaction is the most powerful interaction in the universe. As all motions are spherical, so all dimensional geometry come under the domain of spherical geometry. So spherical geometry of unified force can 'describe everything in the universe' (Hawking, 1996). But unified force does not combine all partial theories but it depends on as all motions are spherical and all motions are alike (Vide: Partial theories and Unified force).

The elementary particles are virtual particles which are not detected by metal detectors, but the natural phenomena caused by virtual particles are visible. 'The force-carrying particles exchanged between matter particles are virtual particles. We know they exist, however, because they have a measurable effect they give rise to forces between matter particles' (Hawking, 1996). Thus we have the results in our hands in favor of unified force such as planetary motions of planets, spiral motions of galaxies, interaction between virtual particles and three matter particles and all motions are alike. Spherical motion of solar system is shown in figure-1. 


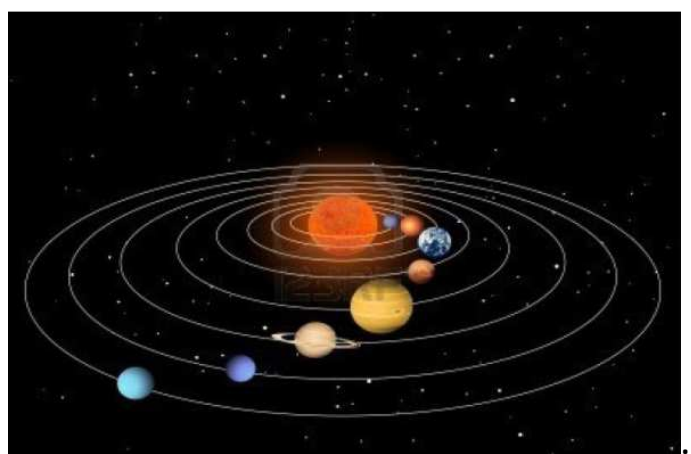

Figure 1. Spherical motion of solar system.

\section{Super Physics}

We have mentioned above that mankind knocks the door of super physics. We may call the new discipline as super physics because this works beyond the range of nuclear physics. 'Nuclear physics deals with nuclei and the release of atomic energy'. We study nuclear physics and taking the advantages of nuclear physics but the technology of super physics is still somehow beyond the reach of mankind. Three basic forces of matter particles such as electromagnetic, weak and strong nuclear interactions belong to nuclear physics. Thus three matter forces may be called planetary forces and have interactions in nucleus such as electromagnetic force shows electromagnetic induction in between electron and proton and electromagnetic wave in short distance, and weak and strong nuclear forces 'binds particles together into combination' (Hawking, 1996). The fourth category is known as gravitational force interacts with three matter forces which belong to super physics. The gravitational force has a number of synonyms according to the nature of works such as elementary forces, long range particles, particles of spin $1 / 2$ and spin $1 / 3$, and making up the matter particles. 'We know they exist, however, because they do have a measurable effect: they give rise to forces between matter particles' (Hawking, 1996). The unified force also belongs to super physics. We know electricity derives from electromagnetic force, in the same way three matter forces derive from unified force. Elementary forces of super physics reign in the universe but the matter forces of nuclear physics reign in the planets. So the technology of the two is different. The technology of super physics is immense important for wellbeing of mankind.

\section{Spiral Motion of Galaxies}

We observe thousands of stars peeps in the sky at night. As it seems, the sky is studded with star or as if a fair of stars in the sky. 'There are some hundred billion $\left(10^{11}\right)$ galaxies, each with, on the average, a hundred billion stars. In all the galaxies, there are, perhaps as many planets as stars, $10^{11} \times 10^{11}=10^{22}$ ten billion trillion (Sagan, 1980). 'Our modern picture of the universe dates back to only 1924, when the American astronomer Edwin Hubble demonstrated that ours was not the only galaxy. There were many others...that can be seen using modern telescope' (Hawking, 1996). All the galaxies have spiral motion among themselves that creates a greater unified force, and the solar unified force is a part of that greater unified force. The greater unified force follows either the principles of vertical or horizontal interaction not both at a time to avoid collision and over crossing each other. Every spherical motion is a unified force in its periphery otherwise it will not work. Here the question of distribution of energy is related. All stars of galaxies have rotational and orbital motion for their in-built nature. Fiery stream blows in spherical way on the surface of the stars that causes the rotational motion of the stars and horizontal interaction to each other causes orbital motion of stars in galaxies. The spiral motion has a chain links and none can break the order. They earn both charges and they select who will earn what kind of charge. A picture of spiral motion of galaxy is shown here in figure- 2 .

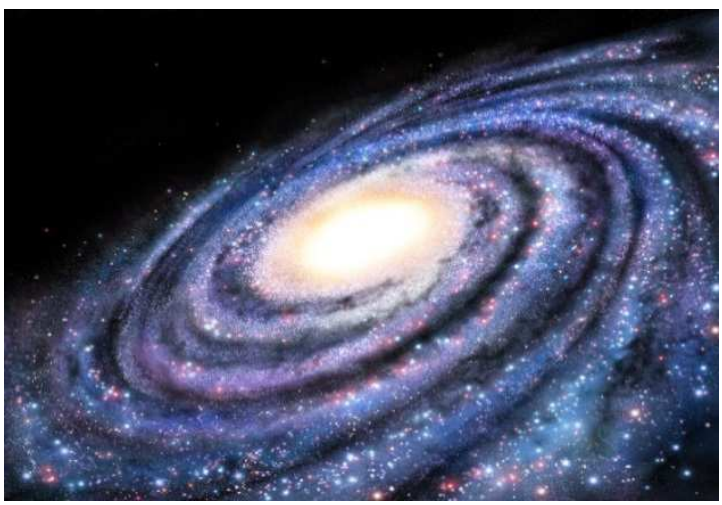

Figure 2. Spiral motion of galaxy.

\section{Partial Theories and Unified Force}

We have a belief that the universe is governed by systemic laws; otherwise the universe would be chaotic and ultimately would be destroyed. But we find that the universe is progressing and expanding smoothly, 'the universe could not be static as everyone previously had thought, but is in fact expanding' (Hawking, 1996). How it is possible? Is this the partial theories which are discovered by our physical sciences in day today life govern the universe? Sometimes it is told that 'you ultimately have to combine the partial theories into a complete unified theory that will describe everything in the universe' (Hawking, 1996). Our partial theories are so diversity that they cannot be combined together keeping their positions all right. Drawing unified force it is found that some of the existing theories may be accepted and some of them may be avoided. Actually our physical sciences started from Greece and gradually came across the mediaeval age, classical age, theoretical age, quantum age, relativistic age and nuclear age. Gradually a lot have been changed and the rest requires to be changed in the light of unified force which is discussed in previous articles published in AJPA by the authors. This has been a factor hindrance to advancement of physical sciences in the level of super physics. But we observed that planets have spherical motion, galaxies have 
spiral motion, wheels of vehicles, motors and motions move in spherical way. It is proved in the previous articles published that all motions are spherical in nature. The main motto in unified force is that all motions are spherical and all motions are alike. Thus we conclude that partial theories may not be combined under unified force but it is spherical motion on which the unified force works.

\section{How Unified Force Works}

Unified force works in support of spherical motion. Both of them are spherical in nature and have the same advantages. In the case of unified force, the long range forces interact with short range three forces lying in inertia of mass of the earth and attract the total frame work of the earth to move around the sun. In the case of spherical motion of bus, train and motor where the vapor of engine gives pressure to the wheel from high pressure to low pressure techniques and the total body moves in spherical motion. Motion also becomes spherical if it passes from vacuum to air, from slow speed to high speed or from motion to rest and vice-versa (Born, 1962). Wind, cyclone, tornado, hurricane, typhoon etc. blow from high pressure to low pressure techniques. Unified force works in the form of spherical motion in magnetic attraction, electromagnetic induction, gravitational field, application of force and refraction of light and mechanical advantages. Unified force works in small scale as well as in large scale with different names. Wheel of vehicles, lever, pulley, crane, mechanical advantages, motions of small periphery are the examples of small scale unified force. Planetary motions and spiral motions of galaxies which are the examples of large scale unified force. All motions are spherical in nature and all motions are alike. Thus we see unified force works in spherical motion, in different fields, in different ways, in different names but in the same technique. How unified force influences the motion is depicted in figure- 3 where the great velocity the path traversed in one second is great, thus the graph line has only a small inclination to the x-axis (3a), the smaller the velocity, the steeper the graph. A point that is at rest has zero velocity. If a point starts as rest and then suddenly acquires a velocity and moves on with this velocity, the graph is broken line one part of which is inclined, the other being vertical(Fig.3b). Similarly broken lines represent cases in which a point that is initially moving uniformly to the right or to the left suddenly changes its velocity (Fig.3c, 3d, 3e) (Born, 1962). How motion becomes spherical when it passes from vacuum to air, from slow speed to high speed or from motion to rest is shown in figure-3.

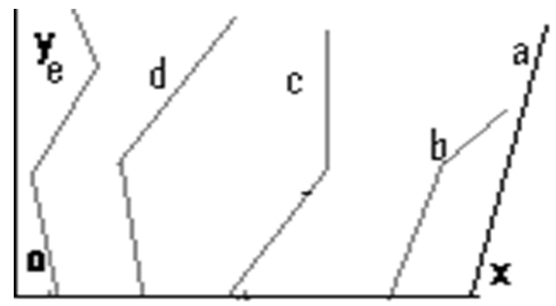

Figure 3. Motion becomes spherical when changes speed.

\section{Force-Carrying Particles}

'Force-carrying particles can be grouped into to four categories according to the strength of the force that they carry and the particles with which they interact' (Hawking, 1996). Here four categories of forces are discussed:

\subsection{Gravitation as a Basic Force}

Gravitation is the basic force in general but the rest three forces are planetary forces. Gravitation is structure less and virtual particles but other three forces are structured form and work in nucleus. Thus gravitation group is the basic forces of elementary particles and the three planetary forces are matter particles work with nucleus, nucleon and atoms. So long mankind knows that gravitation controls the universe and the present research informs us that gravitation creates unified force interacting with three matter forces and that unified force controls the universe. Gravitation is the basic force that controls the universe interacting with matter forces of the planets but three matter forces remain in the nucleus of planets and work absorbing gravitation. Elementary particles are responsible for gravitational force but it's a virtual particle. Gravitation is a mass less and long range force but the rest three are massive and short range matter forces lying in the nucleus of atom of inertia of masses of the earth. Gravitation is created when the long range force and short range force interacts each other. The gravitational interaction is sometime $10^{40}$ weaker than the electromagnetic interaction and the weakest of all. In Newtonian law of gravitation the attraction from distance is weak and attraction from near is strong. There is nothing to be surprised if gravitation attraction is weak from distance. There is no vacuum or empty space in the universe but gravitational force is everywhere. Gravitation as a long range force interacts with short range three matter forces lying in inertia of masses and creates unified force. 'Every particle feels the force of gravity, according to its mass or energy. Gravitation is the weakest of the four forces by a long way; it is so weak that we could not notice it at all were it not for two special properties that it has: it can act over large distance, and it is always attractive' (Hawking, 1996). Sun controls the solar system and it is the source of abundance of energy. The sun rotates on its own axis and at the same time disperses long range particles that induce to the matter forces of distance planets and creates unified force. The atom has at least three phases of energy and they have interaction capacity in their own way but gravitation may not include in it because it is dispersed from the sun and other stars. It may be so happen that the sun and planets disperse how much energy outside, they absorb the same in spherical motion in galaxy, otherwise how they will continue their position with ever expanding and ever lasting universe. In the discussion we shall find that single group of particles are not responsible for gravitation, a number of particles such as spin $1 / 2$ and spin $1 / 3$ of quark groups are responsible for gravitation. 


\subsection{Electromagnetic Force}

'The electromagnetic interaction is responsible for the forces that control atomic structure, chemical reaction and all electric phenomena. It functions only between charged particles, but unlike the gravitational interaction, can be either attractive or repulsive. The interactions is either visualized as a classical field of force analogous to the gravitational field, or as exchange of virtual photons a more convenient form for quantum theory consideration' (Goldstein, 1999). We have conceived that gravitation interacts with three matter forces and the interaction does not mean electric charge or electromagnetic interaction as it is thought in classical mechanics but it means that gravitational particles absorb in the attractive matter particles. In the case of gravitation there is no repulsive force, otherwise gravitation will not work and planets would not move around the sun. Thus the concept of gravitational interaction is separate from the concept of electrical charge or electromagnetic interaction. 'Paulis's exclusion principle says that two similar particles cannot exist in the same state' (Hawking, 1996). In electromagnetic charge, force between two positive charges is repulsive, as is the same between two negative charges, but the force is attractive between a positive and a negative charges. But electromagnetic interaction works in short range of atom only, how can it reach the high space? The wave of gravitational particles reigns there. We are to conclude that in high space gravitational wave sparks light because elementary particles have the capacity but in planet level electrometric interaction and electric charges can emit light. Even stick of matches kindles fire, so they have some differences and similarities. Thus we see that electromagnetic force has internal interaction within the periphery of atom. The gravitation disperses elementary particles in the form of wave in the space what we learn from quantum mechanics. Now-a-days the electronic devices such as wireless, transistor, television, and self phone use magnetic devices to receive the frequencies from transmitter which does not mean that electromagnetic force works everywhere. In high space, gravitational wave reigns. The velocity of light is equal to the velocity of photon as $300000 \mathrm{~km} / \mathrm{s}^{2}$. The spin of lepton, quark and photon is $1 / 2,1 / 3$ and 1 respectively, and smaller the spin more the velocity. Thus lepton and quark groups have two times and three times more velocity than the velocity of photon respectively (Vide: Maxwell's electromagnetic wave).

\subsection{Weak Atomic Force}

The third category is called the weak nuclear force. The weak interaction is $10^{10}$ times weaker than the electromagnetic interaction and responsible for radioactivity and acts on lepton groups of spin $1 / 2$, but not on particles of spin 0,1 and 2, such as photons and gravitons. Physicists suggested that in addition to photon, there were other spin 1 particles, known collectively as massive vector bosons that carried the weak force. 'In the current classification two main classes of particles are recognized; leptons (electron, muon, neutrinos and tau particles) which interact either by the electromagnetic interaction or the weak interaction and have no apparent internal structure; and hadrons (nucleons, pions etc.) which interact by the strong interaction and have a complex internal structure' (Goldstein, 1999).

\subsection{The Strong Nuclear Force}

'The strong interaction, some $10^{2}$ times stronger than the electromagnetic interaction, functions only between hadrons and is responsible for the force between nucleons that gives the atomic nucleus its great stability. It operates at very short range inside the nucleus as little as $10^{-15}$ meter and is visualized as an exchange of virtual masons' (Goldstein, 1999). It holds the quarks together with the proton and neutron and holds the protons and neutrons together with the nucleus of an atom. It is thought that this force is carried by another spin-1 particle. 'The strong nuclear force has a curious property called confinement: it always binds a particle together into combination' (Hawking, 1996).

\section{Gravitational Cycle}

Gravitation works in spherical motion, otherwise planets would not move around the sun. We have spherical motion like water cycle, air cycle, vehicles' cyclic motion, wheels' cyclic motion, electromagnetic cyclic motion etc. Water turns to vapor and vapor turns to cloud and cloud turns to rainfall and water again. It is called water cycle. The current of ocean creates island in the ocean and the current of air creates tornado, hurricane, storm etc in atmosphere. In the same way gravitation has cycle in the space that creates new stars with elementary particles. This is gravitational cycle which has several stages. According to steady state theory, 'new galaxies were continually forming in the gaps in between, from new matter that was being continually created' (Hawking, 1996). Four categories of forces have separate interaction such as electron is responsible for flow of electricity, particles like neutrino maun and tau which interacts either with the electromagnetic interaction or weak interaction, and nucleon and pions which interact with the strong interaction (Goldstein, 1999). Gravitation in the form of elementary forces works as a strong agent with planetary forces and in high space with spiral motion of galaxies. It has similarity with solar energy though it's an inferior force. Thus gravitation may supply powerful energy to mankind for their uses in needs including spacecraft in high space and other fields which will last longer than electricity. This reminds us that gravitation is a great force.

\section{Electromagnetic Induction}

Coulomb first discovered the law of electricity. Ohm, Gauss and others worked on electricity. Faraday became famous for his discovery on electromagnetic induction. 'Faraday discovered that if a magnet is passed through a closed loop of wire, a current will flow in the wire while the magnet is moving. This effect is called electromagnetic induction' (Hart, 1989). He discovered dynamo that produces 
electricity. He also discovered the motor that produces electromagnetic power for the wellbeing of man kind. But he could not put forth electromagnetic law. Because time was not ripped for the purpose. There is a proverb that whatever man discovered, can explain long after. It is unfortunate that Faraday was not considered the inventor of electromagnetic force. We have the same example that Riemann was not considered as pioneer of spherical geometry. Reason is also there as science and mathematics did not advance at that level.

\section{Maxwell's Electromagnetic Wave}

Maxwell used the four laws of electricity and magnetism and predicted that energy of electric and magnetic fields can be propagated in the form of waves, called electromagnetic waves, the velocity of which was calculated and found to be equal to the velocity of light, (Vasudeva, 1983). According to Maxwell's equation electricity and magnetic force interacts with each other and propagates electromagnetic waves all over the world. 'The electromagnetic attraction between negatively charged electrons and positively charged protons in the nucleus causes the electrons to orbit the nucleus' (Hawking, 1996). As we know the electromagnetic force has short range arises in atom and in real field the range of electromagnetic interaction in the coil form may be extended up to mere a centimeter around the coil of motor, how it can propagate electromagnetic wave in high space and all over the world. It is gravitational waves that carry all frequencies all over the world. On the other hand in high space there is no matter particles except gravitational waves of elementary particles (Vide: Where exist four categories of forces). The bigger forces such as the stars and the planets disperse elementary particles in the space in the form of gravitational wave for their high velocity of motion. Maxwell developed electromagnetic wave theory depending on ether theory but afterward ether theory was discarded by Einstein and at the same time electromagnetic wave theory in high space was also cancelled where gravitational field along with wave reigns. Thus we find Maxwell's prediction about electromagnetic wave in high space is failed. According to Pauli's exclusion principle 'two similar particles (forces) cannot exist in the same state' (Hawking, 1996). Here it is stated that electromagnetic wave and gravitational wave cannot exist and work simultaneously in the same region. As regards photon that spread light in apace is a creation of gravitational wave. How gravitational wave moves is shown in figure-4.

\section{elecelunuta}

Figure 4. Gravitational wave in high space.

\section{Principles of Four}

In the second decade of 1920s three giants namely Heisenberg, Schrodinger, and Dirac developed quantum mechanics into a new shape based on the uncertainty principle. According to this theory, particles no longer had separate position and velocity to be observed, instead, they had a combination of position and velocity. Thus quantum mechanics introduces principles of uncertainty or randomness into science. A principle of uncertainty has duality between waves and particles in quantum mechanics. This is known as principle of duality. Einstein contradicts about principle of duality saying that universe was not governed by chance factor and he has a well-known statement 'God does not play dice'. As time passes and knowledge is exploded, principles of duality have expanded and possess a four principle known as particle of position, velocity, force and energy. Now it is principles of four. When we mention long range particle or short range particle and gravitational force we mean particle as a force. No doubt, when one principle is focused, other principle is less focused or willingly suspended for the time being. In other words, the more accurately one will measure one principle of the particle, the less accurately he will measure the other three principles. As the field of particle is so wide that it is difficult to determine four principles simultaneously, so the question of simultaneity does not arise. Example, when the particle exists, it is position, when it moves a long distance or short distance or works with nuclear force or elementary force, it is force, when it works with electromagnetic force or gravitational force, it is wave and every particle has the power of energy. Thus a particle has at least four principles. In the beginning of the nineteenth century our scientists were not much aware of quality of particles, now as we know. The principle of four deals with at least four aspects of same particle namely position, wave, force and energy. The four principles derive from the same source and they work jointly for the creation of unified force.

\section{Where Exist Four Categories of Forces}

'Force-carrying particles can be grouped into four categories according to the strength of the force that they carry and the particles with which they interact... Ultimately, most physicists hope to find a unified theory that will explain all four forces as different aspects of a single force. Indeed, many would say this is the prime goal of physics today. Recently, successful attempts have been made to unify three of the four categories' (Hawking, 1996). That single force is presented here as unified force where four categories of forces have been unified together under the banner of unified force. As we mentioned earlier that gravitation as a long range force discharges from stars and interacts with three matter forces lying in inertia of mass of the earth and other planets and creates unified force. We also mentioned earlier that three planetary forces such as electromagnetic, weak and 
strong nuclear forces lie in the nucleus of atom of inertia of masses of the earth. Now the question arises how and why the three matter forces exist in the inertia of masses of the earth? We discussed earlier that those particles having no structural formation, remain in the sun because there is excessive heat where nucleus, atom or any kind of formative body cannot exist (Vide: Composition of stars). After big bang, the planets detached from the sun and scattered away to a sustainable distance as that of mentioned in Friedmann model and gradually became cool in the atmosphere of planets. Thus the particles of the earth gradually get new formation and shape into nucleus and atoms suitable to different elements and these are still continuing. Among the three planetary forces 'electromagnetic force functions only between charged particles, but unlike the gravitational interaction, can be either attractive or repulsive' (Goldstein, 1999). 'The electromagnetic attraction between negatively charged electrons and positively charged protons in the nucleus causes the electrons to orbit the nucleus of the atom' (Hawking, 1996). The weak and strong nuclear forces 'bind

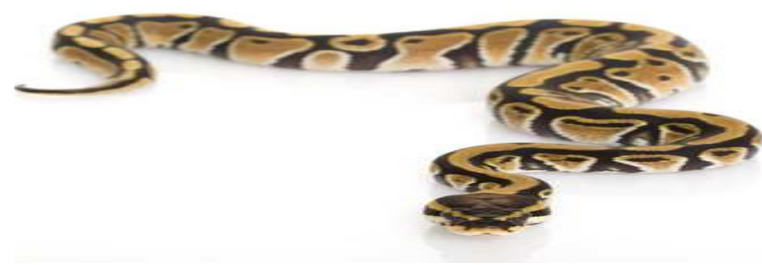

Figure 5. A python camouflages in jungle for prey.

Three planetary forces have very short range inside the atom and have no ability to hunt prey to quench their thirst (interaction) from distance, so they lie in atom in their own position as that of python in the jungle in the hope of they will get something. It is not only python, other beast of prey take position at the time of hunting prey. Nature has both sympathy and cruelty! Long range forces dispersed by the sun when reach the three planetary forces, they immediately grasp the long range forces what we call interaction. Thus we see that electromagnetic force has interaction within the periphery of atom but how can it disperse gravitational wave in high space? The sun and the stars disperse elementary particles in the form of wave in the space what we know as principles of uncertainty. Now-a-days the electronic device such as wireless, transistor, television, and self phone use electromagnetic devices to receive the frequencies from transmitter which does not mean that electromagnetic force works everywhere. Now we find that Maxwell's prediction has failed and electromagnetic wave is not possible in high space where electromagnetic elements such as ethers or nuclei and atoms do not exist (Vide; Composition of stars). In high space, gravitational wave reigns and disperses and receives all frequencies of wave and light all over the world. The gravitation has equal access to three planetary forces that have short range lying in atoms in inertia of masses of the planets. The position of three planetary forces in the nucleus is very meaning full and purposive. particles together into combinations' (Hawking, 1996). Thus we find that the three planetary forces operate at a very short range in a narrow spherical space of nucleus, work tremendous for maintaining relation with the sun and keeping the earth substance together. They have no performance in high space. The performance of three planetary forces may be compared with a sweet metaphor of a python that lies flat in the jungle and pretends as dead to deceive the animals and preys the junior animals when they try to cross over it and swallows at ease. A python cannot chase animals quickly for its slow motion of bulky body, this is why; it takes camouflage and lies flat as dead in the jungle to deceive the animals. When the junior animals like rabbit, fox, jackal etc. try to cross the python thinking it a dry wood, the python immediately grasps it and gradually swallows. The prey begins to cry long time in the mouth of python for his painful death. How a python lies as dead in the jungle shown in figure- 5 and how it preys a beast when it tries to cross the python shown in figure- 6 .

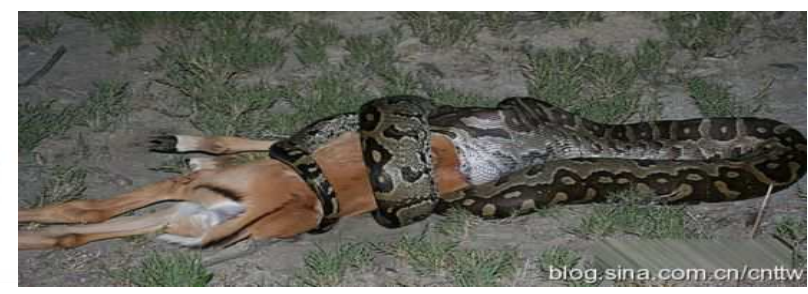

Figure 6. The python devours the prey.

\section{Composition of Stars}

Our scientists think about the phenomena of the universe as they find without following any ultimate norm. There was no ultimate norm so long to follow for any observation of physical sciences. Therefore, their thinking was not sometime absolutely right. Sometime their thinking have been changed. Suppose the idea of black holes changes frequently. We have an old idea that in the core of the sun, hydrogen is converted to helium by nuclear fusion, releasing energy in the process. This idea hampers the systemic thinking and also the progress of unified force as planets are suitable for the growth of nucleus, atoms, molecule, fauna and flora. The composition of stars has been assumed through systematic way in consonance with unified force. As the temperature of the stars is too hot, the elementary particles may not form nucleus and atom in the centre of stars. The question of formation comes in the case of planets, not in the case of stars. The leptons group particles have no structure that exist in the stars, but hadrons group particles have internal complex structure exist in the planets. Particles that have no internal structure lie in the stars and particles that have internal structure lie in the planets. The stars discharge its elementary resources for the wellbeing of the planets, and the planets grasp the resources for spherical motion, developing its ever grown species and material resources. We cannot think that the core of the sun is in gaseous form from 
where planets are scattered. Therefore it is better to think that the core of the sun is in the solid form of elementary particles and they come out due to nuclear fusion. The nuclear fusion is possible in the stars because elementary particles have both charges in their own way. As the elementary particles have charges, so they interact in planetary level. This is the proof that elementary particles have charges in the sun. The electric charges give us electricity and produce nothing, but in the case of elementary particles they produce nucleus and develop atoms adding new electron in the body of atom and thus produce matter. Growth of nucleus is possible in the atmosphere of planet of somewhat cool environment. Thus the planets have suitable environment where the elementary particles are to be formed into nuclei, atoms and matters. We get elementary particles from uranium-235 through high temperature of nuclear fission in nuclear reactor. Einstein formulated $\mathrm{E}=\mathrm{mc}^{2}$ expressing how solid matter changes into elementary particles. But the stars are exception where nuclear fusion is possible through interaction by elementary particles to elementary particles. We are to think about stars in the principle of stars and planet in the principle of planets; otherwise there will be a great wrongness in our assumption of stars and planets. As two giants follow separate principles, they can not follow the same principle. One governs and other is governed. If we conceive existence of hydrogen and helium in stars, it would be a great blunder because star is of burning luminary that always discharges volcanic eruption. On the other hand planets have calm, somber and cold environment suitable to grow and form matters and to live fauna and flora there. If we conceive stars having atoms like hydrogen and helium, it will loose its originality and credibility of hotness and gradually it will be a cool planet and loose its controlling power over the planets and it will cease to and end. 'All the known particles in the universe can be divided into two groups: particles of spin $1 / 2$, which make up the matter in the universe, and particles of spin 0,1 and 2, which we shall see, give rise to the forces between the matter particles' (Hawking, 1996). Here gravitation has synonyms as long range particles and spin $1 / 2$ particles. In the core of the stars, there remain different types of elementary particles in solid form due to high pressure and high temperature and they erupt up by nuclear fusion. When the hot elementary particles come in touch of comparatively cool atmosphere of the earth, form nucleus and hydrogen cell. We know that in high temperature matter breaks up and in cold temperature matter is composed of. The long range particles reach the atmosphere of the earth, develop nucleus and form hydrogen atom. We know 'every particle feels the force of gravity according to its mass or energy' (Hawking, 1996). Thus the hydrogen and other airy atoms absorb one after other elementary particles and forms different types of atoms suitable to 112 elements. Thus the long range particles interact with three planetary forces lying in atom of the inertia of mass of the earth and create unified force. Thus the long range particles make bondage between the stars and the planets, and also between long range particles and short range particles and help the stars to control the planets. The bondage means friendship between two opposite charges as it happens in flora and fauna in the earth. This is the control of the planets from distance. The sample of bondage is made between long range forces and three planetary forces as it happens in the life of couples in planetary life is shown in figure-7.

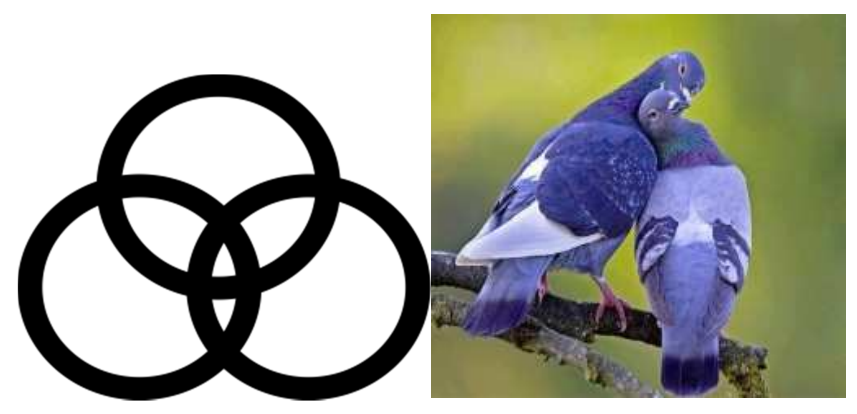

Figure 7. Elementary forces and matter force make bondage as that of a pair of dove in conjugal life.

We know elementary forces discharging from the sun reaches the earth and this force is grasped by three planetary forces. The symbols of three planetary forces are tortoise as electrometric force, bird as weak nuclear force and fox as strong nuclear force. How elementary particles make bondage with three matter particles is shown in figure- 8 .

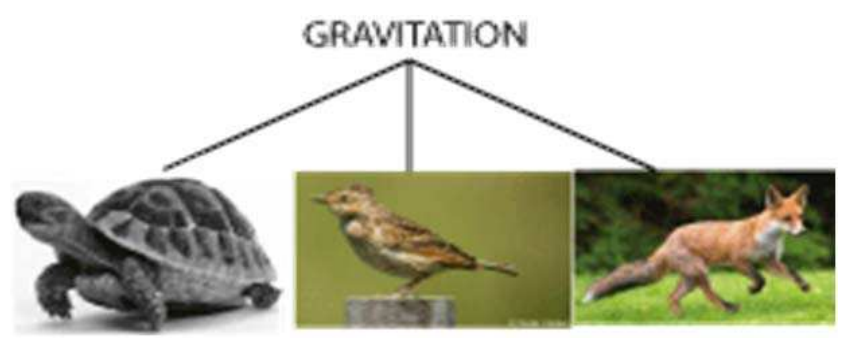

Figure 8. A tortoise, a bird and a fox represent three matter forces on the earth who receive elementary forces.

\section{Non-structured and Structured Particles}

Stars are composed of elementary particles which are indivisible particles. This may be called also fundamental particles. They are imagined as non-structured particles that 'can not be subdivided' (Hawking, 1996). They are conceived as virtual particles that the metal detector cannot identify them. The stars discharge the elementary particles that work as long range forces and control the universe. 'In the current classification two main classes of particles are recognized; leptons (electron, muon and tau particles) which interact either by the electromagnetic interaction or the weak interaction and have no apparent internal structure; and hadrons (nucleons, pions etc.) which interact by the strong interaction and have a complex internal structure' (Goldstein, 1999). 'The force-carrying particles exchanged between matter particles are said to be virtual particles because, unlike real particles, they cannot be directly detected by a particle detector. We know they exist, however, because they do have a measurable 
effect: they give rise to forces between matter particles. Particles of spin 0.1 , or 2 do also exist in some circumstances as real particles, when they can be directly detected (Hawking, 1996).

Planets are composed of structured particles which are known as matter particles. Three basic forces such as electromagnetic, weak and strong nuclear forces is developed in matter particles. The matter particles grow and develop in the planets with the help of gravitation particles and they are divisible. They are short range and massive particles and their jurisdiction is in the periphery of atom. The elementary particles reign all over the world and induce to the three basic forces of atoms in the planets. The elementary particles in the form of gravitation interact with three matter forces in two ways. First, gravitation interacts with three matter forces and summation of three interactions creates unified force and there will be an equation of interaction of four categories of forces that helps to form final equation. Second, unified force would create final equation that works spherical way which grows gradually stronger and controls the universe and all motions. The final equation is $\mathrm{ds}^{2} \infty 2 \pi \mathrm{r}$ that means spherical motion is proportional to circumference by radius (diameter). This equation measures all motions with the help of circumference of spherical motion by radius. Summation of interactions of three basic forces will go to the credit of unified force. The long range forces move spherically and interact with short range forces and create unified force that controls the universe and all motions of the world (Tajuddin and Biswas, 2015).

\section{Elementary Forces and Matter Forces}

We are to classify main forces broadly into two groups such as elementary forces and matter forces. The stars are composed of elementary particles which is a great force that controls the universe. The stars are of very hot, so the elementary particles can not form any kind of structure of particles in the stars. The

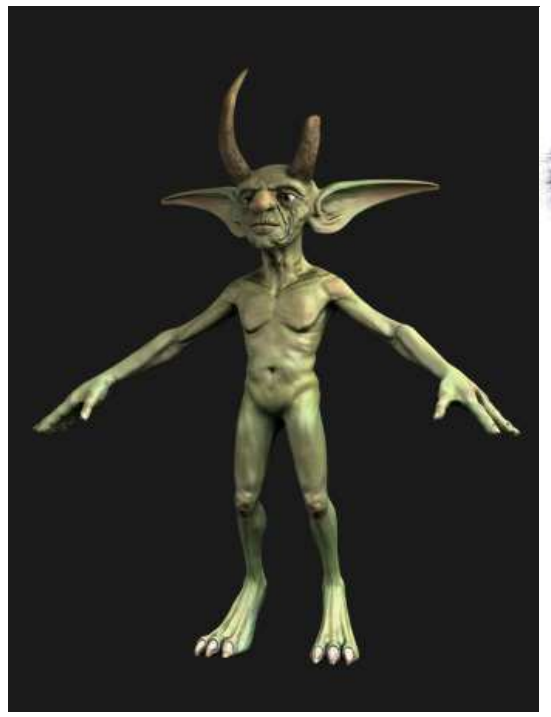

Figure 9. Elementary forces as Ariel. elementary particles can form structure in the planets only. The elementary particles may be called either long range particles or gravitational particles. Thus gravitation as a basic force discharged from the sun and other stars interacts with three matter forces of inertia of mass of planets.

On the other hand matter particles are of three kinds such as electromagnetic, weak and strong nuclear forces. We are to know the distinction among three matter forces. They are massive particles, remain in the atoms of earth and composed of matter particles and their range is very short in between atoms only. The weak and strong nuclear forces are so called binder because they combine the matter in the nucleus of atom. In electromagnetic force, electron moves around the proton of nucleus, and maintains electromagnetic induction with matter forces. Electricity and magnetic force work together in electromagnetic force. These three forces cannot work without the induction of elementary forces. Elementary forces interact with different types of matter forces such as electron, proton, neutron, atom etc. maintaining their separate power, status and existence. Otherwise 'they would all collapse to form a roughly uniform, dense soup' (Hawking, 1996).

Readers might have understood the necessity of two groups of forces. These forces should not be presented in such a way to the readers that these may go beyond the imaginations of the readers and physics may be difficult for them. We are to present two groups of forces with two similes keeping with the nature of their works. Two forces may have similes with two characters of the play The Tempest of the great playwright Shakespeare. Elementary forces may be compared with the giant Ariel and matter forces with Caliban of the drama. Readers may have two imageries in their mind as elementary forces with Ariel who plays his role with sorcery, and matter forces with Caliban who is an ugly animal inferior to human being seems coming out of earth but works hard for island dwellers. Here the role of elementary forces played by Ariel is shown in figure 9 and a matter forces by Calliban in figure 10 .

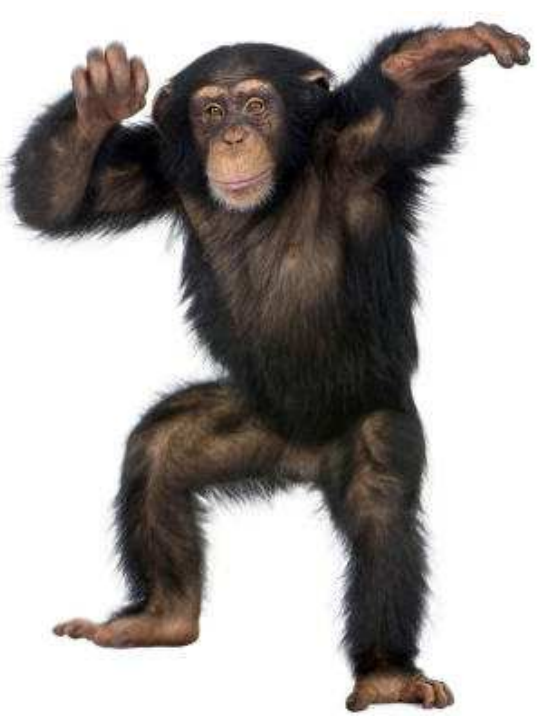

Figure 10. Matter Forces as Caliban. 


\section{Problems of Elementary Particles}

We have an old idea that in the core of the sun, hydrogen is converted to helium by nuclear fusion, releasing energy in the process. This idea hampers systematic thinking and also the progress of unified force and super physics because planets are suitable for the growth of nucleus, atoms, molecule, fauna and flora. The composition of stars has been assumed in systematic way in consonance with unified force. As the temperature of the stars is too hot, the elementary particles may not form nucleus and atom in the centre of stars. The question of formation of structure comes in the case of planets, not in the case of stars. The leptons group particles have no structure that exist in the stars, but hadrons group particles have internal complex structure exist in the planets (Goldstein, 1999).Particles that have no internal structure lie in the stars and those who have internal structure lie in the planets. The stars discharge its elementary resources for the wellbeing of the planets, and the planets grasp the resources to build its ever grown species and material resources. It is assumed that the elementary particles themselves remain in the solid form in the core of the stars and they come out due to nuclear fusion. The nuclear fusion is possible in the stars because elementary particles have both charges in their own way. As the elementary particles have charges, so they interact in planetary level. This is the proof that elementary particles have charges in the sun and other stars.

\section{Acquiring the Technology of Elementary Forces}

Mankind approaches to acquire the technology of matter forces such as electricity, electromagnetic, weak and strong nuclear forces. In this purpose mankind developed battery cell, dynamo, generator and hydroelectric project, solar energy project and nuclear reactor for producing and using electricity in different electronics devices, and different types of motors in electromagnetic devices, and different plants for nuclear devices. Producing electricity and its growing usages has become cottage industry in human society. But mankind is far behind in acquiring and using the technology of elementary forces. Now-a-days human race may not be satisfied with the present speed of traveling and other facilities available to them which has become back dated such as locomotive trains, slow motion of plane even snail's pace rocket and in other fields in their life of high expectancy. 'One cannot travel faster than light means that it would take far too long to travel between stars and galaxies' (Hawking, 1996).Mankind desires to reach the highest level by using the highest technology of elementary forces of super physics in all levels of physics, chemistry and biological sciences. Thus it is necessary to increase the capacity of energy producing machineries and plants to the highest level and to find out the gap of present capacity and desired goals to the top most level. What probability the elementary forces show us, to sustain that capacity for utilization is also important. Mere discovery of any source of elementary forces without sustaining the capacity-build and utilizing the capacity will not bring credit to the wellbeing of mankind.

\subsection{How Nuclear Reactor Works}

'A nuclear reactor in which a nuclear fission, chain reaction is sustained and controlled in order to produce nuclear energy, radioisotopes or new nuclides. The fuel available for uses in a fission reactor are uranium-235, uranium-233, and plutonium-239, only the first occurs in nature, the others have to be produced artificially. When a uranium-235 nucleus is made to under fission by the impact of a neutron it breaks into two roughly equal fragments, which release either two or three very high energy neutrons. These fast neutrons need to be slowed down to increase the probability that they will cause further fissions of ${ }^{235} \mathrm{U}$ nuclei and thus sustain the chain reaction. This slowing down process occurs naturally to a certain extent when the neutrons collide with other nuclei, unfortunately, however, the predominant uranium isotope, ${ }^{238} \mathrm{U}$, absorbs fast neutrons to such an extent that in natural uranium the fission reaction is not self-sustaining. In order to create a controlled self-sustaining chain reaction it is necessary either to slow down the neutrons (using a moderator in a thermal reactor) to greatly reduce the number absorbed by ${ }^{238} \mathrm{U}$, or to reduce the predominance of ${ }^{238} \mathrm{U}$ in natural uranium by enriching it with more ${ }^{235} \mathrm{U}$ than it normally contains. In a fast reactor the fuel used is enriched uranium and no moderator is employed.

In thermal reactors, neutrons are slowed down by collisions with light moderator atoms (such as graphite, deuterium, or beryllium), they are then in thermal equilibrium with the surrounding material and are known as thermal neutrons. In a heterogeneous thermal reactor the fuel and moderator are in separate solid and liquid phases (e.g. solid uranium fuel and a heavy water moderator).In the homogeneous thermal reactor are mixed together, for example in a solution, molten dispersion, slurry, or suspension.

In the reactor core the fuel elements encase the fuel; in a heterogeneous reactor the fuel elements may fit into a lattice that also contains the moderator. The progress of the reaction is controlled by 'control rods' which when lowered into the core absorb neutrons and so slow down or stop the chain reaction. The heat produced by the nuclear reaction in the core is used to generate electricity by the same means as in a conventional power station, i.e. by raising steam to drive a steam turbine that turns a generator. The heat is transferred to the steam-raising boiler or heat-exchanger by the coolant. Water is frequently used as the coolant; in the case of the boiling water reactor (BWR) and the pressurized reactor (PWR) water is both coolant and moderator. In the BWR the primary coolant raises steam in a secondary circuit for driving the turbine. In the gas-cooled reactor the coolant is a gas, usually carbon dioxide with as outlet temperature of about $350^{\circ} \mathrm{C}$, or $600^{\circ} \mathrm{C}$ in the 
case of the advanced gas-cooled reactor (AGR).

In fast reactor, in which there is no moderator, the temperature is higher and the liquid-metal coolant is used, usually liquid sodium. Some fast reactors are used as converters or breeders. A converter reactor is one that converts fertile material (such as ${ }^{238} \mathrm{U}$ ) into fissile material (such as ${ }^{239} \mathrm{Pu}$ ). A breeder rector produces the same fissile material as it uses. For example, a fast breeder reactor using uranium enriched with ${ }^{239} \mathrm{Pu}$ as the fuel can produce more ${ }^{239} \mathrm{Pu}$ than it uses by converting ${ }^{238} \mathrm{U}$ to ${ }^{239} \mathrm{Pu}$ '(Goldstein, 1999).

\subsection{Nuclear Fission and Fusion}

'In nuclear fission reactions a neutron is used to break up a large nucleus, but in nuclear fusion the two reacting nuclei themselves have to be brought into collision. As both nuclei are positively charged there is a strong repulsion force between them, which can only be overcome if the reacting nuclei have very high kinetic energies. These high kinetic energies imply temperatures of the order of $10^{8} \mathrm{k}$ ' (Goldstein, 1999). Unification theory differs with existing idea of nuclear fusion (vide: Composition of stars).

\subsection{Findings and Utilizations}

So far we have discussed about different types of nuclear reactors, nucleus fission and fusion as to how neutrons collide with nuclei through chain reaction and sustain the reaction by moderator for producing nuclear energy, radioisotopes or new nuclides. What we find above may be summarized in support utilization under unification theory as follows: First, it is noted that in the case of nuclear fusion and fission, there is similarity and dissimilarity as both require high temperature but the temperature is higher in nuclear fusion in the stars. Second, nuclear fission is man-made, works in controlled environment using moderator but nuclear fusion works in free environment of the sun and the stars. Third, in fast fission when the neutrons collide with other nuclei, unfortunately, the predominant uranium isotope, ${ }^{238} \mathrm{U}$, absorbs fast neutrons to such an extent that in natural uranium the fission reaction is not self-sustaining. Fourth, when a uranium-235 nucleus is made to under fission by the impact of a neutron it breaks into two roughly equal fragments, which release either two or three very high energy neutrons. This development may be called increase of matter particles by the interaction of long range forces and short range forces in the earth and planets. Fifth, when the fast fission happens, the neutrons collide with other nuclei in natural uranium, predominant uranium isotope absorbs fast neutrons and breaks into two fragments that hampers the chain reaction, and now the question arises as to absorption of neutron by nuclei and breaking into two equal fragments, what resembles in nuclear fusion where the long range forces interact with short range forces in atoms of the earth and creates numerous atoms. Sixth, chain reaction happen in nuclear fission unless it is controlled by using moderator, but due to nuclear fusion in the sun, the long range particles dispersed a long way and interact with short range forces in atoms of the earth and chain reactions in atoms create further atoms continually in the earth. Seventh, nuclear fast fission is controlled by using slow fission by using moderator such as graphite, deuterium and beryllium or by the liquid-metal coolant, usually liquid sodium in order to produce nuclear energy, radioisotopes or new nuclides. Eighth, chain reaction in fast fission may not be continued because of increased high temperature that may cause blast the reactor and also causing the damage of man and material. Ninth, alternative plant is devised which is working under ground in Jura Mountain near Geneva in Switzerland to collide electron with proton or vice versa to find out the smallest particles, and that was happened in 2012 where smallest particle was produced named as God-particle. Tenth, nuclear reactor can facilitate two types of technologies with the same ends: one end leads to producing nuclear energy, radioisotopes and new nuclides; second end leads to producing nuclear weapons and the technology how to change and utilize the old bombs into energy. Eleventh, now the question arises how to preserve the God-particles in some sort of storage by using some sort of moderator with a view to achieving and utilizing the highest level of technology cherished by mankind in bosom. Twelfth, atom possesses 'some 200 short lived elementary particles', each of which possesses separate capacity and radioactivity; which to be searched and utilized for the wellbeing of them. It is important to know how the method of thermal reactor can harness a source of energy for men's use. The stories of finding and using elementary particles have been told but we are to progress further. 'How dull it is to pause, to make an end, to rust unburnished, not to shine in use' (Tennyson). Present processes of nuclear plants are to be checked where lies the gap to reach the perspective goal of elementary particles the highest level of aspiration for the wellbeing of mankind. Geek hero Ulysses says 'I shall see life to the lees' (Tennyson).

\section{Mathematical Analysis}

Three matter forces such as electromagnetic, weak and strong nuclear forces have own equations and strength but now the equation and strength of unified force such as interaction of gravitation with three matter forces must be greater than any of the forces. The summation of strength of three matter forces will be greater than any of the forces. Unified force also creates new horizon in the field of nuclear forces and elementary forces and as a result a number of new equation will be added and a number of old equation will be discarded.

\subsection{Spherical Geometry of Unified Force}

We already have three categories of coordinates that may be transformed to spherical motion adding time with them. First, Riemann's transformation of three dimensional Cartesian coordinates ( $\mathrm{x}, \mathrm{y}, \mathrm{z})$ into N-dimensional co-ordinates, second, transformation of Cartesian coordinates $(\mathrm{x}, \mathrm{y}, \mathrm{z})$ into polar coordinates $(r, \theta, \phi)$, and third, Einstein's metric tensor. These three coordinates are transformed from 
linear to spherical coordinates and hence they have limitations for many reasons and may not be applied in the field properly.

Examples of the coordinates:

1) N-dimensional spherical tensor: $\mathrm{ds}^{2}=g_{\mathrm{ijk}} d \mathrm{x}^{\mathrm{i}} \mathrm{dx^{ \textrm {j } }} d \mathrm{dx}^{\mathrm{k}} \mathrm{dt}^{2}$ $(\mathrm{i}, \mathrm{j}, \mathrm{k}=1,2,3,4)$

2) Three dimensional Cartesian coordinates $(\mathrm{x}, \mathrm{y}, \mathrm{z})$ into $(r$, $\theta, \phi)$ as Polar spherical tensor:

Example: $d s^{2}=d r^{2}+r^{2} d \theta^{2}+r^{2} \sin ^{2} \theta d \varphi^{2}$

3) Einstein worked with the traditional system of geometry that was development with method of Bianchi Identities. This tensor turns to zero tensor of four dimensions joining one of time with three of space. Einstein commits the same fallacy what his predecessors faced. Perhaps Einstein did it without his knowing mind because he was not aware of spherical motion of four dimensions, and of time if it is added with space, it would revolve. His tensor was also defective as he used zero tensor in his metric. Actually time remains separated in his practice. Coordinates of four dimensional coordinates is shown in figure -11 .
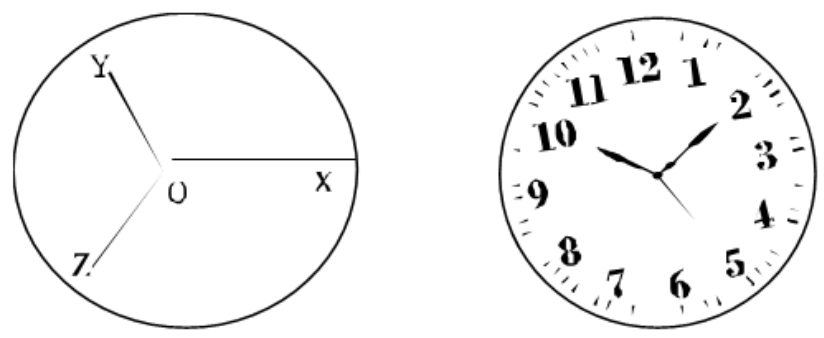

Figure 11. Four dimensional coordinate by Einstein

Example: Einstein's four dimensional metric:

$\mathrm{ds}^{2}=-\mathrm{dx}^{2}-\mathrm{dy}^{2}-\mathrm{dz}^{2}+\mathrm{c}^{2} \mathrm{dt}^{2}$ (Vide: AJPA 2015; 3(5)166-177)

\subsection{Unified Law}

Non-Euclidean two dimensional spherical geometry was developed by Gauss and others and subsequently three dimensional geometry by Riemann in coordinate system. Schwarzschild developed external solution using polar coordinate. But spherical geometry of unified force is developed by the first author depending on spherical motion supported by four properties of matter such as radius of circle, mass of the body, cyclic frequency and time. Here coordinate system is abandoned. Thus spherical geometry of single dimension is developed as line element for unified force. It has validity, objectivity and usability. It is a single law that may be applied in all laws of applied force or law of gravitation. It has supremacy over three previous mentioned spherical coordinates (Vide: AJPA, 2015; 3(3)69-76). The line element of unified force is $\mathrm{ds}^{2} \infty 2 \pi \mathrm{r}$ that has three explanations. First, main formula ( $\mathrm{ds}^{2} \infty 2 \pi \mathrm{r}$ ) in literary is spherical motion is proportionate to circumference by radius. Second formula is applied in the field (Vide: 19.3) and third formula is comparative formula (Vide: 19.4). Explanation of line element of unified law is shown in table-1
Table 1. Line element of unified law.

\begin{tabular}{ll}
\hline $\mathrm{Ds}^{2} \propto 2 \pi \mathrm{r}$ & (Main formula) \\
$\mathrm{ds}^{2} \propto 2 \pi \mathrm{r}=$ p. $2 \pi \mathrm{r}$. sf. dt & (Usable formula) \\
$2 \pi \mathrm{r} \propto 2 \mathrm{r}$ & (Comparative formula) \\
\hline
\end{tabular}

Here,

$\mathrm{ds}^{2}=$ Line element

$2 \pi \mathrm{r}=$ Boundary of unified force

$2 \mathrm{r}=$ Radius as working force

$\mathrm{p}=$ Inertial force/unified force taken as constant

$\mathrm{sf}=$ Spherical /cyclic frequency

$\mathrm{dt}=$ Time

If a motion curves every moment, it stands to spherical motion and gradually it becomes circle. How a round body moves easily in spherical way is shown in figure- 12 .

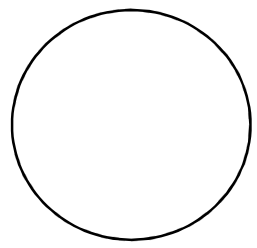

Figure 12. Spherical motion of round body.

\subsection{How Unified Force Works}

The idea of equation is taken from Radian of Trigonometry where circumference $=2 \pi \mathrm{r}$ ( circumference $/$ diameter $=\pi$, or, circumference $=\pi d=2 \pi \mathrm{r}$ ). Here the concept $2 \pi \mathrm{r}$ represents the boundary of the spherical motion whose radius ' $r$ ' controls the boundary. In the formula, $2 \pi \mathrm{r}$ means spherical motion of unified force and ' $r$ ' means radius that controls spherical motion of unified force. Inertial mass and gravitational mass both are considered as constant because inertia derives from a gravitation which is a constant force(Vide: AJPA, 2015; 3(3)69-76). Example: how five elements work in spherical motion of unified force is shown in figure 13.

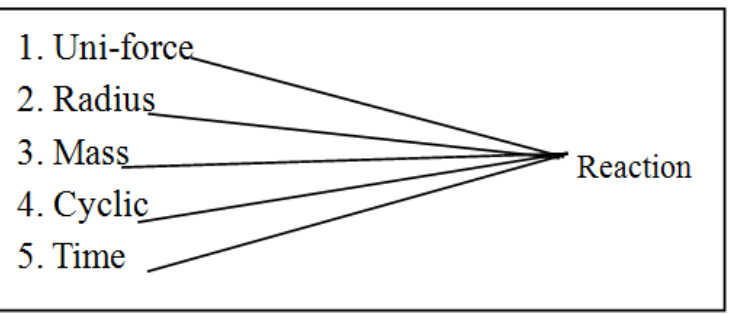

Figure 13. Unified force works with five properties of matter.

\subsection{Comparison of Unified Force and Linear Force}

In comparative equation, motion in gravitational field is compared with that of non-gravitational field. Here a motion in gravitational field moves $2 \pi \mathrm{r}$ (circumference) in a circle within a fixed time, that motion in non-gravitational field moves equivalent to $2 \mathrm{r}$ (diameter) of that circle. The motion is linear and uniform in non-gravitational field but that of in gravitational field is spherical and accelerated. Newtonian's laws of motion are linear and uniform but unified motion is spherical and accelerated. This comparison is shown in figure 14. 


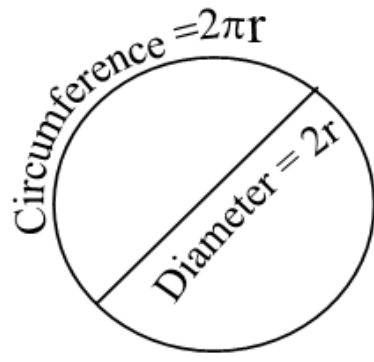

Figure 14. Comparison between linear and spherical motion.

\section{Conclusion}

The universe has single ultimate principle known as unified force on which the universe is working. The unified force is deep rooted to the formation of the universe. The elementary particles spherically discharged from the distance, interact with three matter forces such as electromagnetic, weak and strong nuclear forces lying in the inertia of masses of the planets and create unified force that controls the solar system and the same type of greater unified force controls the universe. After big bang the universe scattered away and the break away parts began to move spherical way to keep a linkage among them. Galaxies used to move in spiral motion, and planets moved around the stars. Thus all motions come under domain of unified force. Thus the universe is not chaotic but cosmos. The elementary particles are virtual particles which are not detected by metal detectors, but the natural phenomena caused by virtual particles are visible. 'The force-carrying particles exchanged between matter particles are virtual particles. We know they exist, however, because they have a measurable effect they give rise to forces between matter particles' (Hawking, 1996). Thus we have the results in our hands in favor of unified force such as planetary motions of planets, spiral motions of galaxies, interaction between virtual particles and matter particles and all motions are alike.

\section{References}

[1] Einstein, A. (1961) Relativity: The Special and the General Theory, Three Rivers Press, New York.

[2] Goldstein, A. M. (1999), A Dictionary of Physics, CBS Publishers \& Distributors, New Delhi, P82, 180.

[3] Hawking, S. W. (1996), A Brief History of Time, Bantam Books, New York, London, PP. 67-84, 86, 42.

[4] Kindersley, D. (2003), Ultimate Visual Dictionary, $21^{\text {st }}$ Century Supplement, London, New York, P. 32.

[5] Born, M. (1962), "Einstein's Theory of Relativity", Dover Publications Inc., New York.

[6] Prakash, S. (1985), Relativistic Mechanics, Pragati Prakashan, Meerut, India, P, 363.

[7] Rashid, A. M. H. (1984), Einstein \& The Theory of Relativity, Bangla Academy, Dhaka, P. 98.

[8] Strathern, P. (1997) Einstein and Relativity, Arrow Books, London, United Kingdom, P. 78.

[9] Sagan, C. (1980) Cosmos, Ballantine Books, New York, P.1, 3.

[10] Shakespeare, W. (1564-1616)The Tempest, one of his plays.

[11] Tajuddin, $\mathrm{M}$ and Biswas, M. H. A. (2015), Einstein: Unification Theory and Unification of Basic Forces: published by American Journal of Physics and Application, New York, 2015;3(3)69-76.

[12] Tajuddin, M and Biswas, M. H. A. (2015), Einstein: Unification Theory and Unification of Basic Forces: published by American Journal of Physics and Application, New York, 2015; 3(5) 166-177.

[13] Tajuddin, M. Biswas, M. H. A. and Islam, A. Review of the Theory of Relativity, Journal of Applied Sciences Research, 4(1):32-39,200.

[14] Tennyson, L. A. (1809-1892), Ulysses, one of his best poems.

[15] Vasudeva, D. N. (1983), Fundamentals of Magnetism and Electricity, S. Chand \& Company Ltd. New Delhi, P.583.

[16] White, M. and Gribben, J. (2005). "A Life of Science: Einstein", p. 249, The Free Press, London, UK. 